\title{
The Relationship between Uric Acid and Metabolic Parameters in Hypotyroidic Patients
}

\author{
Mehmet Mustafa Gülddï ${ }^{1}$, Okan Akyüz ${ }^{2}$, İsa Ardahanlı ${ }^{3}$ \\ ${ }^{1}$ Department of Internal Medicine, Istanbul Mehmet Akif Ersoy Thoracic and Cardiovascular Surgery \\ Training and Research Hospital, Turkey \\ ${ }^{2}$ Department of Nephrology, Bilecik State Hospital, Turkey \\ ${ }^{3}$ Department of Cardiology, Bilecik State Hospital, Turkey.
}

*Corresponding Author: Mehmet Mustafa Güldü, Mehmet Akif Ersoy Thoracic and Cardiovascular Surgery Training and Research Hospital, Istanbul, Turkey.

\begin{abstract}
Introduction: Thyroid hormones play an important role in almost all metabolic events in the body. Historically, the relationship between uric acid levels and thyroid diseases has been a matter of curiosity. Our aim in the study was to investigate how uric acid metabolism is affected in hypothyroid patients.

Materials and Methods: Patients diagnosed with hypothyroidism were included in the study. It was divided into two groups, as an apparent and subclinical hypothyroid group. Patients who were treated with the diagnosis of hypothyroidism were divided into two groups as TSH targeted and non-targeted. Participants without the disease and drug use affecting uric acid levels were designed as a control group. The groups included in the study were compared both among themselves and with the control group.

Results: The number of female patients in the hypothyroid group was statistically significant compared to the control group $(p<0.001)$. As expected, TSH levels were significantly higher and fT4 significantly lower in the patient group ( $p<0.001$ and $p<0.001$, respectively). There was no significant difference in uric acid values in both groups $(p<0.05)$. No statistically significant difference was found between subgroups of patients with hypothyroidism and when the control group and serum uric acid levels were compared ( $p$ $<0.05)$.
\end{abstract}

Conclusions: We think that the thyroid dysfunction of the patient should be known to correctly evaluate the laboratory parameters that can be measured. More extensive randomized controlled trials are needed for this.

Keywords: Hypothyroidism, uric acid, metabolic parameters

\section{INTRODUCTION}

Thyroid hormones play an active role in the metabolic activities, development, and growth of all tissues in the body. Therefore, the insufficiency of these hormones affects many systems. At rest, stroke volume and heart rate decrease. As a result, cardiac output decreases peripheral vascular resistance increases, and hypertension are observed $(1,2)$. Also, renal blood flow, glomerular filtration rate, tubular secretion, and reabsorption are reduced. The kidneys' ability to concentrate urine may be slightly impaired and mild proteinuria may be seen (2). Two basic mechanisms are thought to play a role in the physiopathogenesis of the increase in serum uric acid levels associated with hypothyroidism. These are an increase in uric acid synthesis due to increased purine metabolism and / or a decrease in excretion of uric acid. It was concluded that the increase in serum uric acid values associated with hypothyroidism was not due to overproduction, but to a decrease in the clearance of uric acid (3). There are a limited number of studies in the literature evaluating the relationship between uric acid and hypothyroidism, which is thought to be an important marker in the evaluation of cardiovascular and renal prognosis, especially recently. The purpose of this retrospectively planned study is to evaluate the serum uric acid levels in patients with hypothyroidism under initial diagnosis and treatment (3).

\section{Materials AND Methods}

Our study was carried out between January 2010 and May 2012 at Haydarpaşa Numune Training and Research Hospital in Turkey. Patients with 
newly diagnosed, apparent hypothyroidism, subclinical hypothyroidism and previously treated with the diagnosis of hypothyroidism were retrospectively screened. The control group was selected from individuals who applied to the internal medicine clinic, who had no known disease and who did not find a disease as a result of their examination. Venous blood was collected from the groups included in the study after at least eight hours of fasting. fT3, fT4, thyroid-stimulating hormone (TSH), blood urea nitrogen (BUN), creatinine, and uric acid values were recorded retrospectively. Biochemical samples were measured by the photometric method in the Biochemistry Laboratory of Haydarpaşa Numune Training and Research Hospital by using the Abbott Architect C16000 autoanalyzer. The participants were divided into 5 groups.

Group 1: Newly diagnosed patients with undiagnosed hypothyroidism (Patients without any thyroid disease or history of thyroid surgery or diagnosed and not treated for at least 8 weeks) (fT4 $<0.60 \mathrm{ng} / \mathrm{dL}$ and TSH level $>10$ $\mathrm{mIU} / \mathrm{L}$ )

Group 2: Newly diagnosed patients with subclinical hypothyroidism (No history of thyroid disease or thyroid surgery, sT3 and sT4 values are between normal values ( 0.60 to 1.64 $\mathrm{ng} / \mathrm{dL}$ ) and TSH level> 4mIU / L)

Group 3: Patients previously diagnosed with hypothyroidism, under thyroid hormone replacement therapy for at least 6 weeks, with no $\mathrm{TSH}$ value on target $(\mathrm{TSH}>2.5 \mathrm{mIU} / \mathrm{L})$

Group 4: Patients previously diagnosed with hypothyroidism, under thyroid hormone replacement therapy for at least 6 weeks, with TSH value on target (TSH value $0.60-2.5 \mathrm{mIU} / \mathrm{L}$ )

Group 5: The control group was formed from healthy volunteers whose fT3, fT4, and TSH levels were within normal limits and who did not use any drugs.

Exclusion criteria of the study:

1. Having chronic liver disease

Table1. Demographic characteristics of the groups
2. With known gout and urate nephropathy

3. Pregnant women and women planning a pregnancy within a year

4. Patients with malnutrition

5. Patients who have undergone a surgical operation recently (in the past three months)

6. Known coronary artery disease

7. Those with known malignancy

8. <18 age and > 65 age

9. Patients receiving thyroid hormone replacement therapy and having $\mathrm{TSH}<0.5$ $\mathrm{mIU} / \mathrm{L}$

10. Patients using agents that may affect uric acid level (losartan, hydrochlorothiazide, cyclosporin, tacrolimus, allopurinol, ascorbic acid, theophylline, warfarin, ethambutol, benzbromarone, colchicine, furosemide, probenecid, febuxostat) were not included in the study.

\section{Statistical Analysis}

SPSS 23 (Statistical Package for Social Sciences-SPSS) was used in the analysis of the research. In addition to the deterministic statistics, a one-sample t-test was used in the average value comparison of the patient and control groups, and the One-Way ANOVA (Variance analysis) test in the average comparison of the binary groups. The analysis was used to evaluate the relationships between the parameters. The results were evaluated with $95 \%$ confidence interval and $p<0.05$ significance level.

\section{RESULTS}

A total of 151 patients and 69 control groups taken between the ages of 18-65 were included in the study. The mean age of the patient group was $44.90 \pm 12.16$, and the mean age of the control group was $42.37 \pm 12.82$. There was no significant difference in age between the patient and control groups $(p=0.161)$. Female patients were statistically higher in the patient group (84.1\% vs $49.2 \%$; $p<0.001$ ) (Table 1 ).

\begin{tabular}{|l|l|l|l|}
\hline & Group with hypothyroidism & Control group & p values \\
\hline $\mathrm{n}(\%)$ & $151(68.6 \%)$ & $69(31.4 \%)$ & \\
\hline Age (years) (mean $\pm \mathrm{SD})$ & $44.90 \pm 12.16$ & $42.37 \pm 12.82$ & 0.161 \\
\hline Female & $127(84.1 \%)$ & $34(49.2 \%)$ & $<\mathbf{0 . 0 0 1}$ \\
\hline Male & $25(15.9 \%)$ & $35(50.8 \%)$ & $<\mathbf{0 . 0 0 1}$ \\
\hline
\end{tabular}

Thirty patients $(19.8 \%)$ in the group with apparent hypothyroidism, $28(18.5 \%)$ in the subclinical hypothyroid group, $62(41.05 \%)$ in the TSH non-target group and $31(20.5 \%)$ in the TSH target group were included. Patient subgroup distribution is shown figure 1 . 
The Relationship between Uric Acid and Metabolic Parameters in Hypotyroidic Patients

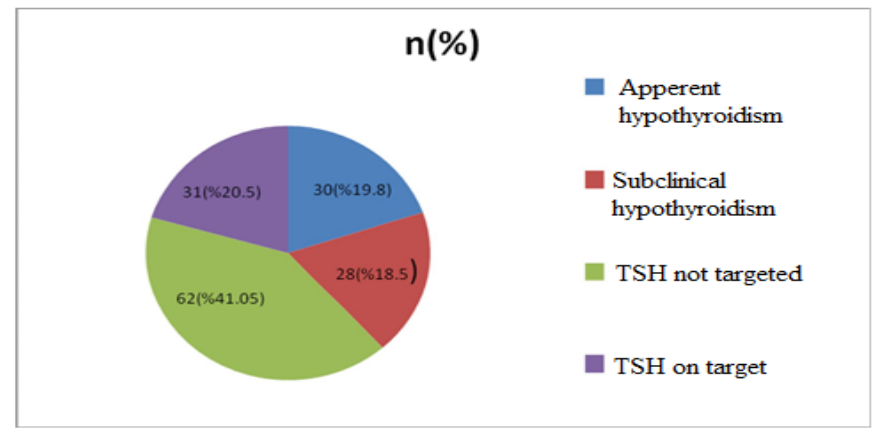

Figure1. Subgroups of patients with hypothyroidism

In basal thyroid function tests comparison, TSH and FT4 values were statistically significantly higher in the patient group ( $\mathrm{p}<0.001)$.

In the biochemical analysis, mean fasting glucose values in patients with hypothyroidism were statistically significantly higher than the control group $(\mathrm{p}<0.001)$. Triglyceride and LDL values were higher in the hypothyroid group than in the control, but this difference was not statistically significant $(\mathrm{p}=0.050$ and $\mathrm{p}=0.010$, respectively). Hemoglobulin values were lower in the hypothyroid group than the control group, but this difference was not statistically significant $(\mathrm{p}=0.050)($ Table 2$)$.

Table2. Comparison of basal laboratory values of the groups

\begin{tabular}{|l|c|c|c|}
\hline & Hypothyroidism Group & Control Group & P value \\
\hline $\mathrm{n}$ & 151 & 69 & $<\mathbf{0 . 0 0 1}$ \\
\hline Glucose (mean $+\mathrm{SD})$ & $99.65 \pm 33.68$ & $82.54 \pm 10.75$ & 0.221 \\
\hline BUN (mg / dL) & $12.35 \pm 3.9$ & $13.1 \pm 4.07$ & 0.375 \\
\hline Creatinine (mg / dL) & $0.70 \pm 0.15$ & $0.73 \pm 0.13$ & 0.388 \\
\hline ALT (U / L) & $18.7 \pm 10.8$ & $20.4 \pm 11.4$ & 0.598 \\
\hline AST (U / L) & $19.9 \pm 10.1$ & $20.8 \pm 8.0$ & 0.651 \\
\hline Uric Acid (mg / dL) & $4.48 \pm 1.63$ & $4.58 \pm 1.34$ & 0.010 \\
\hline LDL (mg / dL) & $128.7 \pm 41.5$ & $111.0 \pm 27.9$ & 0.050 \\
\hline Triglyceride (mg / dL) & $142.4 \pm 97.0$ & $108.1 \pm 69.0$ & 0.582 \\
\hline White Blood Cells (mm $\left.{ }^{3}\right)$ & $7.1 \pm 1.7$ & $6.9 \pm 1.6$ & 0.050 \\
\hline Hb (g / dL) & $12.2 \pm 1.4$ & $13.1 \pm 2.0$ & 0.107 \\
\hline Platelets (mm $\left.{ }^{3}\right)$ & $273.10 \pm 72.18$ & $253.30 \pm 43.59$ & 0 \\
\hline
\end{tabular}

The mean uric acid value in the hypothyroid group was $4.48 \pm 1.63$ and $4.58 \pm 1.34 \mathrm{mg} / \mathrm{dL}$ in the control group. There was no significant difference in uric acid values in both groups $(\mathrm{p}=$ $0.651)$.

Table3. Average uric acid levels of hypothyroid subgroups

\begin{tabular}{|l|l|}
\hline Subgroups & Average Uric Acid (mg / dL) \\
\hline Group 1 (Apparent hypothyroidism) & $5.19 \pm 2.57$ \\
\hline Group 2 (Subclinical hypothyroidism) & $4.11 \pm 1.41$ \\
\hline Group 3 (TSH in non-target) & $4.43 \pm 1.29$ \\
\hline Group 4 (TSH on target) & $4.23 \pm 1.003$ \\
\hline
\end{tabular}

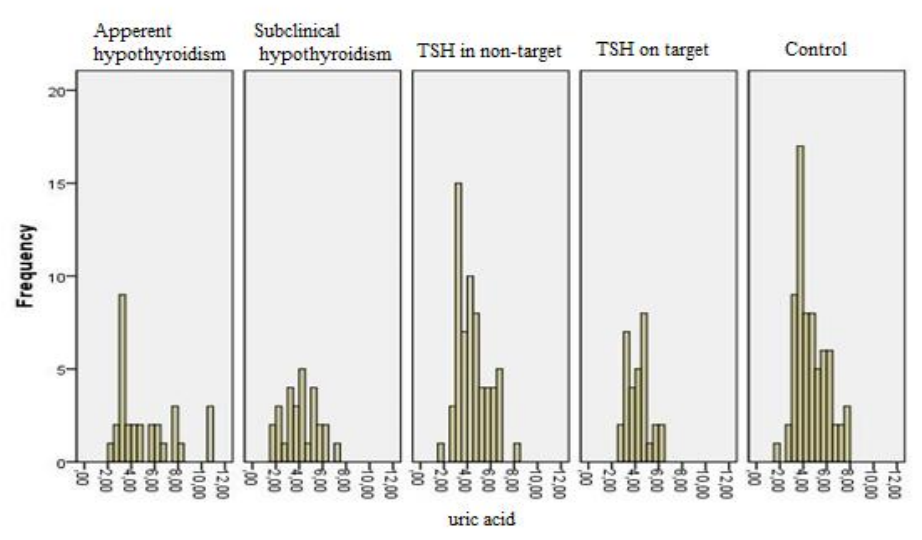

Figure2. Average uric acid levels of subgroups
There was no significant difference between the subgroups in terms of mean uric acid values. The distribution of the average uric acid values of the subgroups is shown in table 3 and figure 2 . 
When the creatinine levels of the subgroups of patients with hypothyroidism were compared, there was no statistically significant difference. (p> 0.005). The subgroups of patients with hypothyroidism are not statistically significant (p>0.005) between the control group and serum uric acid levels.

\section{DISCUSSION}

Thyroid hormones are critical determinants of somatic development in the brain and infants and metabolic activity in adults. They affect almost all functions in metabolism. To perform these functions, thyroid hormones must be in continuous functioning. There are large thyroid hormone stores in the thyroid gland to maintain its functionality. In addition, thyroid hormone biosynthesis and secretion are kept within narrow limits by a regulatory mechanism that is very sensitive to small changes in circulating hormone concentrations.

Uric acid is the final product of the metabolism of adenosine and guanosine based purines, which are released as a result of the catabolism of nuclear material. Purine nucleotides are destroyed by sequential separation of the components that constituent the nucleotides. Since there is no uricase (uric oxidase) enzyme in the human organism, the end product of this catabolism is uric acid. There is no universally accepted definition of hyperuricemia. Evidence supports the idea that hyperuricemia may be a key mechanism in activating the reninangiotensin and cyclooxygenase- 2 (COX-2) systems in progressive renal disease (4).

It is controversial that uric acid is an independent risk factor for heart disease. However, important information has been obtained about the relationship between hyperuricemia, gout and comorbid conditions, especially cardiovascular morbidity and mortality and serum urate levels (5). Studies suggest that thyroid hormones and serum uric acid levels are two important factors that affect especially the cardiovascular system and renal functions. Cellular effects of thyroid hormone mediate the binding of triiodothyronine (T3) to nuclear receptors by binding T3-receptor complexes to DNA, it regulates the expression of genes that regulate the calcium cycle in the cardiac myocyte (6). Hyperuricemia in kidney failure is caused by reduced uric acid excretion and hyperuricosuria is not accompanied.

The heart is the main target for thyroid hormone effect (7). At rest, the stroke volume and heart rate decrease. As a result, cardiac output decreases and peripheral vascular resistance increases and hypertension is observed $(8,9)$. Renal blood flow, glomerular filtration rate, tubular secretion and reabsorption are decreased. Urine concentration ability of kidneys is impaired, proteinuria can be seen (9). But; There are studies evaluating the relationship between uric acid and hypothyroidism, which are thought to be an important marker in the evaluation of cardiovascular and renal prognosis, especially recently. In the absence of thyroid hormones, serum uric acid renal clearance decreases and uric acid levels increase. The reason for this is thought to be reduced metabolic activity.

Besides, pathological glomerular changes such as glomerular basement membrane thickening and mesangial matrix expansion in patients with hypothyroidism contribute to the reduction of renal blood flow. Glomerular filtration rate decreased in $40 \%-55 \%$ of patients with hypothyroidism. In the animal model study conducted by Dariyerli et al. On this subject, it has been shown that glomerular filtration rate decreases and uric acid excretion decreases and serum uric acid levels increase in rabbits endowed methimazole (10). In another study related to this subject, 48 patients with hypothyroidism had thyroid hormone replacement for 6 weeks. It was observed that serum creatinine and uric acid levels decreased and became similar to the control group (11).

In our study, we did not find a statistically significant difference between serum uric acid and creatinine levels in the total hypothyroid group and the control group. In addition, uric acid and creatinine levels of the hypothyroid subgroups were not found to be statistically significant between them and when compared with the control group $(\mathrm{p}=0.651)$. We think that this outcome, which is incompatible with the literature, is due to the low number of subgroup patients in our study. Unlike previous studies, the ages of the total hypothyroid group and the control group were homogeneously distributed in our study. Similar to other studies, the number of female patients was higher in the hypothyroid group compared to the control group ( $\mathrm{p}<0.001)(11)$.

In our study, serum glucose ( $\mathrm{p}<0.001)$, LDL ( $\mathrm{p}$ $=0.010)$ and triglyceride $(\mathrm{p}=0.050)$ levels of patients with total hypothyroidism were found to be statistically significantly higher compared to the control group. This result is compatible 
with the results of other studies investigating the relationship between hypothyroidism and insulin resistance and lipid metabolism in the literature $(12,13)$.

Consequently, the levels of biochemical molecules that can be measured, such as uric acid and other, should be carefully monitored and interpreted in thyroid hormone disorders, as they are affected by both metabolic activity and renal excretion. More comprehensive studies are needed on this subject.

\section{REFERENCES}

[1] Kowalik M. A., Columbano A., Perra A. (2018). Thyroid hormones, thyromimetics and their metabolites in the treatment of liver disease. Frontiers in endocrinology: 9, 382.

[2] Jameson J.L, Weetman A.P. Disorders of the Thyroid Gland in.; Harrison's Principles of internal medicine. 18 th edition Mc Graw Hill Companies, United States (2012): 2911 -2940

[3] Hsieh Y. P., Chang C. C., Yang Y., Wen Y. K., Chiu P. F., Lin C. C. (2017). The role of uric acid in chronic kidney disease patients. Nephrology, 22(6), 441-448.

[4] Kang D. H. (2014). Uric Acid and the Kidney. In Core Concepts in Parenchymal Kidney Disease (pp. 375-388). Springer, New York, NY.

[5] Ariev A. L., Kunitskaya N. A., Kozina L. S. (2013). New data on gout and hyperuricemia: Incidence rates, risk factors and agingassociated manifestations. Advances in Gerontology, 3(2), 138-141.

[6] Klein I., Ojamaa K. (2001). Thyroid hormone and the cardiovascular system. New England Journal of Medicine, 344(7), 501-509.
[7] Grais I. M., Sowers J. R. (2014). Thyroid and the heart. The American journal of medicine, 127(8), 691-698.

[8] Canepa M., Viazzi F., Strait J. B., Ameri P., Pontremoli R., Brunelli C., ... \& AlGhatrif M. (2017). Longitudinal association between serum uric acid and arterial stiffness: results from the Baltimore longitudinal study of aging. Hypertension, 69(2), 228-235.

[9] Elsurer R., Afsar B. (2014). Serum uric acid and arterial stiffness in hypertensive chronic kidney disease patients: sex-specific variations. Blood pressure monitoring, 19(5), 271-279.

[10] Dariyerli N., Andican G., Çatakoglu A. B., Hatemi H., Burçak G. (2003). Hyperuricemia in hypothyroidism: is it associated with postinsulin infusion glycemic response?. The Tohoku journal of experimental medicine, 199(2), 59-68.

[11] Marwah S., Mehta M., Shah H., Haridas N., Trivedi A. (2015). Correlation of serum uric acid and serum creatinine in hypothyroidism. National Journal of Physiology, Pharmacy and Pharmacology, 5(3), 232-235.

[12] See L. C., Kuo C. F., Yu K. H., Luo S. F., Chou I. J., Ko Y. S., ... \& Liu J. R. (2014). Hyperthyroid and hypothyroid status was strongly associated with gout and weakly associated with hyperuricemia. PloS one, 9(12).

[13] Baldwin W., McRae S., Marek G., Wymer D., Pannu V., Baylis C., ... \& Sautin Y. Y. (2011). Hyperuricemia as a mediator of the proinflammatory endocrine imbalance in the adipose tissue in a murine model of the metabolic syndrome. Diabetes, 60(4), 1258-1269.

Citation: Mehmet Mustafa Güldü et.al, "The Relationship between Uric Acid and Metabolic Parameters in Hypotyroidic Patients", International Journal of Research Studies in Medical and Health Sciences. 2020; 5(1): 20-24.

Copyright: (C) 2020 Mehmet Mustafa Güldü et.al,, This is an open-access article distributed under the terms of the Creative Commons Attribution License, which permits unrestricted use, distribution, and reproduction in any medium, provided the original author and source are credited. 